\title{
US GeoData Available Through the Internet
}

The U.S. Geological Survey (USGS) offers certain US GeoData data sets through the Internet. They can be retrieved using the World Wide Web or anonymous File Transfer Protocol (FTP). The data bases and their directory paths are as follows:

- 1:24,000-scale digital line graph data in SDTS format (/pub/data/DLG/24K)

- 1:2,000,000-scale digital line graph data in SDTS format (/pub/data/DLG/2M)

- 1:100,000-scale digital line graph data (/pub/data/DLG/100K)

- 1:100,000-scale land use and land cover data (/pub/data/LULC/100K)

- 1:250,000-scale land use and land cover data (/pub/data/LULC/250K)

- 1:24,000-scale digital elevation data (/pub/data/DEM/7.5min)

- 1-degree digital elevation model data (/pub/data/DEM/250)

\section{World Wide Web Access}

US GeoData files can also be retrieved through a USGS World Wide Web server using standard Web browsers. The following address is for the USGS GeoData home page:

edcwww.cr.usgs.gov/doc/edchome/ $\mathrm{ndcdb} / \mathrm{ndcdb}$.html

Each of the data bases can be navigated alphabetically, by State and (or) through a graphical index. Choose a method of navigation by clicking on that option. Then locate the desired file.

Under the options menu, turn on the "Load to local disc" option.

Click on the file to be downloaded.

\section{FTP Access}

Follow the instructions below to download files from the above data bases using standard anonymous FTP:

- Go to the "pub/data" directory
- Enter the Internet address: edcftp.cr.usgs.gov

- At the user name, enter anonymous

- At the password, enter your e-mail address.

- Please select the type of data you wish to download.

- The files are listed by area name, generally in alphabetical order; for example, the Austin, Tex., quadrangle would be found under the directory "A."

- When the desired quadrangle has been located, set the file transfer mode to binary by entering binary at the prompt. Then enter get and the quadrangle name.

\section{README Files}

README files can be downloaded from the data base directories for information concerning the US GeoData files. It is recommended that users download and read the README file for their desired data base before changing the transfer mode and downloading the quadrangle files.

\section{File Formats}

Data files retrieved using FTP may need to be translated from one format to another to make them usable with different application programs. The US GeoData files, for example, have been compressed with the GNU public domain "gzip" utility. Users without access to gzip can uncompress the file as it is retrieved by leaving off the ".gz" extension.

The gzip program is available for a variety of platforms by means of anonymous FTP at the following sites:

- prep.ai.mit.edu

- enterprise.nwi.fws.gov

Uncompressed files for these data are typically five times larger than compressed versions and therefore will take longer to transfer.

\section{National Geospatial Data Clearinghouse}

Metadata describing the data sets are available in electronic form through the USGS node of the National Geospatial Data Clearinghouse (NGDC). Use the following Internet address for the USGS node: nsdi.usgs.gov/

The USGS continues to add data and metadata to this node in support of the National Spatial Data Infrastructure.

\section{Information}

Specific information concerning the data bases can be found in US GeoData users guides, which are available through anonymous FTP at the following Internet address:

mapping.usgs.gov/www/html/2nmpgds.html/

Read the file "README" in the public directory to find the directory location for the word processing or plain text versions of the appropriate US GeoData users guide. It is recommended that users download and read these files before transferring detailed information.

For further information about Internet access to US GeoData files, contact customer services:

U.S. Geological Survey

EROS Data Center

Customer Services

Sioux Falls, SD 57198

605-594-6151; Fax 605-594-6589

E-mail: custserv@usgs.gov

To purchase a hard copy of any users guide or get information on other USGS products and services, call 1-888-ASK-USGS, use the Ask.USGS fax service, which is available 24 hours a day at 703-648-4888, or visit the general interest publications Web site on mapping, geography, and related topics at mac.usgs.gov/mac/isb/pubs/pubslists/ index.html.

For additional information, visit the ask.usgs.gov Web site or the USGS home page at www.usgs.gov. 